increase PrEP awareness. While urban women did not endorse the same concerns over where they would receive PrEP, they commonly reported concerns that PrEP would lead to increased "promiscuity" among its users.

Conclusion Black women in the Deep South had differing preferences regarding PrEP service delivery and messaging depending on whether they lived in urban or rural settings. These findings can inform targeted intervention development by emphasizing the need for partnerships with trusted community organizations and utilization of service delivery strategies that allow for anonymity to increase uptake of PrEP especially among rural Black women.

Disclosure No significant relationships.

\section{P421 "THE PROMISE OF PREP": MOTIVATIONS FOR TAKING PREP AMONG EARLY-ADOPTING NEW ZEALAND GAY AND BISEXUAL MEN}

Tanushi Punchihewa, Peter Saxton*, Janine Wiles. University of Auckland, School of Population Health, Auckland, New Zealand

\subsection{6/sextrans-2019-sti.507}

Background In 2018 amid rising HIV diagnoses, New Zealand became one of the first countries to fully fund pre-exposure prophylaxis (PrEP) through its public health system. PrEP has clear HIV prevention benefits but also potential trade-offs, namely behavioural risk compensation among gay and bisexual men (GBM). These concerns can trouble public health leadership, soften PrEP promotion and delay service re-orientation towards PrEP delivery, hampering implementation. Understanding the motivations of early PrEP adopters could address stakeholder concerns and improve PrEP roll-out.

Methods We examined data from the baseline "NZPrEP" demonstration project among 150 GBM conducted in Auckland sexual health clinics 2017-18. All participants completed a linked anonymous online survey at enrolment. Open-ended responses to questions on $\mathrm{PrEP}$ motivations and clinic experiences were coded in NVivo and subject to inductive thematic analysis. Secondly, using a deductive approach we applied the identified themes to the theory of planned behaviour (TPB).

Results We identified six motivations by early-adopters for taking PrEP: risky behaviour; engaging in condomless sex; altruism; risk reduction and prevention; early and free access to PrEP; and peace of mind and autonomy. These themes clearly mapped onto the three main tenets of TPB, namely behavioural beliefs, normative behaviours, and perceived behavioural control. In addition, five themes were also identified regarding PrEP implementation: accessibility; clearer communication; greater promotion; clinic attitude; and gratitude.

Conclusion Early-adopters expressed several motivations for PrEP that align with recognised public health values. Themes such as altruism (wanting to protect partners and the community as well as oneself), risky behaviours (difficulties negotiating safe sex) and peace of mind (reducing anxiety in a high HIV prevalence community) can help persuade stakeholders that PrEP is an ethical as well as an effective HIV prevention tool. Such findings are especially pertinent for government officials, general practitioners, the general public and gay communities themselves.

Disclosure No significant relationships.

\section{P422 USE OF DOXYCYCLINE PROPHYLAXIS AGAINST STI AMONG GAY AND BISEXUAL MEN TAKING PRE- EXPOSURE PROPHYLAXIS IN MELBOURNE}

Eric Chow*, Christopher Fairley. Alfred Health, Melbourne Sexual Health Centre, Carlton, Australia

\subsection{6/sextrans-2019-sti.508}

Background Two trials have shown the use of doxycycline prophylaxis could prevent sexually transmitted infections such as chlamydia and syphilis among men who have sex with men (MSM) but its use is controversial because of concerns about the potential to increase antimicrobial resistance. This study aimed to estimate the proportion of MSM who used doxycycline prophylaxis and the related factors.

Methods MSM who taking pre-exposure prophylaxis for HIV (PrEP) attended the Melbourne Sexual Health Centre between June and November 2018 were invited to complete a question on whether they had taken doxycycline to prevent STI in the past month. Demographic characteristics and sexual behavioural data were also collected as part of routine STI care. Multivariable logistic regression with generalised estimating equations was used to identify the factors associated with the use of doxycycline prophylaxis for STI.

Results There were 1,686 men reported taking PrEP during the study period and 1,065 (63\%) completed the additional question on doxycycline. Of those, 105 men $(9.9 \%$; 95\% CI: 8.1-11.8\%) used doxycycline prophylaxis in the past month. Multivariable analysis showed that men who injected drugs in the last 3 months had higher odds of using doxycycline prophylaxis (aOR 3.26; 95\% CI: 1.50-7.08) compared to those who did not inject drugs. Use of doxycycline prophylaxis was not associated with demographic characteristics (age and country of birth) and sexual behaviours (number of casual partners and condomless anal sex in the last 3 months).

Conclusion About one in ten MSM taking PrEP who also use doxycycline prophylaxis to prevent STI. This is the first estimate among Australian MSM and it is similar to the data from London showing 8\% of MSM taking doxycycline prophylaxis in a sample of 106 MSM. Use of doxycycline prophylaxis is associated with drug use behaviours but not sexual behaviours.

Disclosure No significant relationships.

\section{P423 PRE-EXPOSURE PROPHYLAXIS AS AN ALTERNATIVE TO PEP FOR ELECTIVES. SURVEY ASSESSING MEDICAL STUDENTS' KNOWLEDGE AND BELIEFS} ${ }^{3}$ John Mcsorley. 'King's College London, GKT School of Medical Education, London, UK; ${ }^{2}$ King's College London, King's Undergraduate Medical Education in the Community, London, UK; ${ }^{3}$ London North West University Healthcare NHS Trust, Northwick Park Hospital GUM, London, UK

\subsection{6/sextrans-2019-sti.509}

Background Pre-exposure prophylaxis (PrEP) is indicated for many populations at a higher risk of acquiring HIV through sexual exposure. Healthcare students engaging in medical electives may also be at a higher risk of acquiring HIV through occupational exposure. Since access to post-exposure 